\title{
Optimum hovering wing planform
}

DOI:

10.1016/j.jtbi.2016.06.024

\section{Document Version}

Accepted author manuscript

Link to publication record in Manchester Research Explorer

\section{Citation for published version (APA):}

Nabawy, M. R. A., \& Crowther, W. J. (2016). Optimum hovering wing planform. Journal of Theoretical Biology, 406, 187-191. https://doi.org/10.1016/j.jtbi.2016.06.024

\section{Published in:}

Journal of Theoretical Biology

\section{Citing this paper}

Please note that where the full-text provided on Manchester Research Explorer is the Author Accepted Manuscript or Proof version this may differ from the final Published version. If citing, it is advised that you check and use the publisher's definitive version.

\section{General rights}

Copyright and moral rights for the publications made accessible in the Research Explorer are retained by the authors and/or other copyright owners and it is a condition of accessing publications that users recognise and abide by the legal requirements associated with these rights.

\section{Takedown policy}

If you believe that this document breaches copyright please refer to the University of Manchester's Takedown Procedures [http://man.ac.uk/04Y6Bo] or contact uml.scholarlycommunications@manchester.ac.uk providing relevant details, so we can investigate your claim.

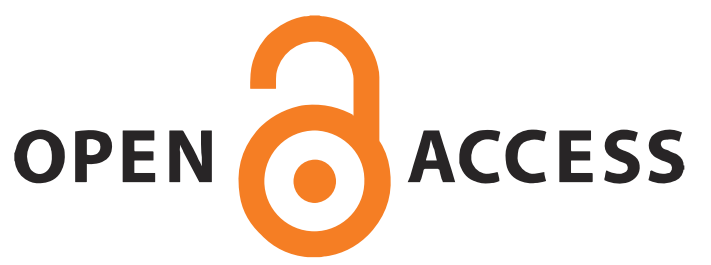




\section{Author's Accepted Manuscript}

Optimum Hovering Wing Planform

Mostafa R.A. Nabawy, William J. Crowther

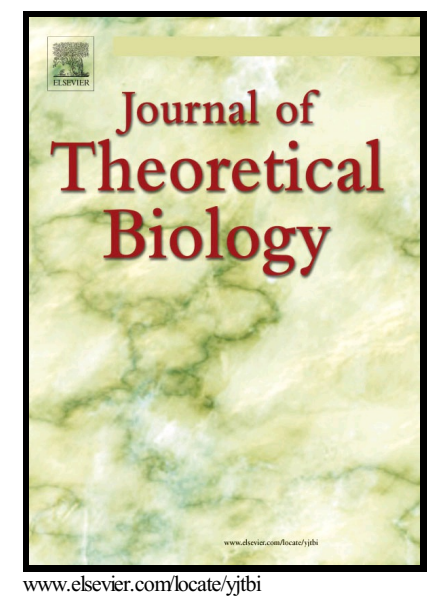

PII:

S0022-5193(16)30158-8

DOI: $\quad$ http://dx.doi.org/10.1016/j.jtbi.2016.06.024

Reference: YJTBI8712

To appear in: Journal of Theoretical Biology

Revised date: 14 June 2016

Accepted date: 18

Cite this article as: Mostafa R.A. Nabawy and William J. Crowther, Optimun Hovering Wing Planform, Journal of Theoretical Biology http://dx.doi.org/10.1016/j.jtbi.2016.06.024

This is a PDF file of an unedited manuscript that has been accepted fo publication. As a service to our customers we are providing this early version o the manuscript. The manuscript will undergo copyediting, typesetting, an review of the resulting galley proof before it is published in its final citable form Please note that during the production process errors may be discovered whic could affect the content, and all legal disclaimers that apply to the journal pertain 


\title{
Optimum Hovering Wing Planform
}

\author{
Mostafa R. A. Nabawy", William J. Crowther \\ School of Mechanical, Aerospace and Civil Engineering, The University of Manchester, Manchester, M13 9PL, UK \\ *Correspondence: mostafa.ahmednabawy@ manchester.ac.uk
}

\begin{abstract}
Theoretical analysis is used to identify the optimum wing planform of a flapping/revolving wing in hover. This solution is of interest as a benchmark to which hovering wing geometries driven by broader multidisciplinary evolutionary or engineering constraints can be compared. Furthermore, useful insights into the aerodynamic performance of untwisted hovering wings are delivered. It is shown that profile power is minimised by using an untwisted elliptical planform whereas induced power is minimised by a more highly tapered planform similar to that of a hummingbird.
\end{abstract}

Keywords: Insect flight, flapping wings, revolving wings, aerodynamics, hummingbird, elliptic wing.

\section{Letter}

Many constraints act on selection of wing planform shape for insects including aerodynamics, physiology and body configuration. The primary constraint is understood to be driven by aerodynamic performance; however, understanding in this area remains incomplete. This study aims to understand the degree to which designs in nature are optimal with respect to aerodynamic performance. In this letter, we introduce a theoretical solution for the optimum planform of hovering wings used to provide weight support by flapping or revolving in a stationary fluid.

There have been several efforts to understand the effect of wing geometry on the aerodynamic performance of hovering wings. Using a non-linear unsteady theoretical model, Ansari et al. [1] varied one wing geometrical parameter at a time to investigate the effect on lift and lift to torque ratio characteristics. The general conclusion is that the best wings are those with nearly straight leading edges and more area outboard. Experimentally, Usherwood and Ellington measured the force coefficients on 
wings with different geometries [2,3]. They found that the coefficients are unaffected by significant alterations in leading edge shape, twist, or camber [2]. They also found a relatively minor effect of the wing aspect ratio on the measured coefficients at incidence angles below 50 degrees [3]. Kruyt et al. [4] revised this through measuring the lift and torque of hummingbird wings of limited aspect ratio range. They found a modest aspect ratio effect on lift and drag characteristics but a significant aspect ratio effect on the power factor. Later, experimental investigations were conducted for hovering rectangular wings of a wide range of aspect ratios [5,6]. The main conclusion is that wings with high aspect ratios (compared to insect wings aspect ratios) operating at high angles of attack stall due to the breakdown of the leadingedge vortex. The interest in investigating the aspect ratio effect on insect-like hovering wings extends to numerical studies as well (e.g. [7]) which showed significant correlation between the flow structure and aerodynamic forces with wing aspect ratio.

The aspect ratio effect has attracted significant attention in recent years; the effect of wing chord distribution (planform) is less understood. Furthermore, there are no studies considering the effect of wing geometry on the profile and induced losses associated with lift generation in hovering flight. Thus, this study identifies an optimum chord distribution for hovering insect-like wing to which other existing hovering planforms can be compared. It also assesses the induced and profile power characteristics of relevant wing planforms for hovering flight.

Aerodynamic force generation from hovering insect-like flapping motion can be divided into a quasisteady aerodynamic mechanism during the wing translational phase and unsteady aerodynamic mechanisms related to stroke reversals (such as wing rotation, added mass and wake capture) [8]. These unsteady mechanisms are known to play an important role within flight control and manoeuvrability [2]. However, for sustained hover, insects typically employ symmetrical half-strokes and the aerodynamic forces required for weight support are adequately accounted for by quasi-steady aerodynamics over the wing translational phase alone $[2,9,10]$. Therefore, in this work, we focus the discussion on the optimum wing planform required within the steady translational phase. For brevity, and in keeping with the insect 
flight aerodynamics literature, we refer to wings within this steady translational phase as 'revolving wings' [2].

The optimum wing is defined as a wing that produces a given lift for the least total power required, where the total power is made up from both profile and induced components [11]; consequently, for a wing to be optimum for a given amount of lift [12]:

(a) Each wing section should be operated at its optimum effective angle of attack to minimise profile power, and

(b) The downwash distribution along the wing length should be uniform to minimise the induced power.

In this study, a quasi-steady linearised aerodynamic treatment is considered. Thus, the local wing lift coefficient, $C_{l}$, is given by:

$C_{l}(r)=C_{l \alpha, 2 d}(\underbrace{\alpha_{g}(r)-\alpha_{i}(r)}_{\alpha_{e}(r)}),(1)$

where $\alpha_{g}$ is the geometric angle of attack, $\alpha_{e}$ is the effective angle of attack and $\alpha_{i}$ is the induced angle of attack defined as:

$\alpha_{i}(r)=w(r) / V(r),(2)$

where $w$ is the downwash velocity, $V$ is the sectional flow velocity and $r$ is the distance along the span from the wing root. Note that for revolving wings the $2 \mathrm{~d}$ airfoil lift curve slope, $C_{l \alpha, 2 d}$, may vary with span as the local Reynolds number varies; however, the assumption of a constant average value along the wing length does not lead to any serious loss of accuracy [12]. In addition, the linearity assumption is not a concern because non-linear lift curves associated with hovering insect-like wings can be conveniently represented using an equivalent linear lift curve representation as detailed in [13].

We first review the optimum design of a wing in parallel translational motion (i.e. fixed wing) for which the sectional flow velocity along the wing length is constant. Minimum profile power for a given amount of lift is achieved when each section of the wing operates at its optimum effective angle of attack. 
Assuming the wing section is constant along the span then the optimum effective angle of attack is constant. The minimum profile power condition can be achieved simultaneously with the minimum induced power condition since the constant downwash required for minimum induced power also gives a constant effective angle of attack. Thus for a practical design solution, a minimum total power wing for a given flight condition (speed, required lift force) can be achieved without wing twist by implementing an elliptic chord distribution to give minimum induced power (constant downwash) and adjusting the wing area such that the required angle of attack is equal to the optimum angle of attack.

Consider now the optimal design of a revolving wing which is the concern of this work. Here, the sectional flow velocity along the wing takes the linear form:

$V(r)=\dot{\phi} r=\dot{\phi} R \hat{r}, \quad \hat{r}=\frac{r}{R}, \quad 0 \leq \hat{r} \leq 1,(3)$

where $\dot{\phi}$ is the angular velocity and $R$ is the length of the wing. Given equations 2 and 3, for a revolving wing with constant downwash distribution, the induced angle of attack distribution will be hyperbolic. Alternatively, if the induced angle of attack distribution is constant, the resulting downwash distribution will be non-constant (linear function of wing length). Thus to achieve minimum total power for a revolving wing, it is necessary to prescribe both the wing planform and the wing twist distribution.

The design process for an optimal revolving wing is as follows. First, the minimum profile power constraint is met by specifying an appropriate geometric twist distribution, $\alpha_{g}(r)$, to achieve a constant effective angle of attack equal to the wing section angle of attack for minimum profile power, $\alpha_{e, o p t}$, for a constant downwash $[12,14]$ :

$\alpha_{g}(r)=\alpha_{e, o p t}+\frac{w}{\dot{\phi} r} .(4)$

It should be noted that typical values for optimum effective angle of attack for helicopter rotors range between 5 and 10 degrees (see Figure 3.9 of [12]). In hovering insect flight, the skin friction contribution to overall drag is higher than it is for rotors operating at higher Reynolds numbers, hence the optimum 
effective angle of attack is typically higher, e.g. between 10 and 30 degrees (for example see [2] and [10]). Next the chord distribution required to achieve the constant downwash is specified in terms of the circulation distribution along the wing and the section lift coefficient required for the minimum profile constraint $[12,15]$ :

$c(r)=\frac{2 \Gamma(r)}{C_{l} V(r)}=\frac{2 \Gamma(\hat{r})}{\left(C_{l \alpha, 2 d} \alpha_{e, o p t}\right)(\dot{\phi} R \hat{r})} \cdot(5)$

In the helicopter literature $[12,14]$, the optimum chord distribution for minimum power is derived from a simple physical interpretation based on momentum theory. The implemented model assumes a constant bound circulation to produce a uniform downwash distribution [12] (which is clearly incorrect, but nonetheless fit for purpose). From equation 5, it thus follows that the optimum chord distribution would be of a hyperbolic shape (due to the linear velocity distribution in denominator). According to the Helmholtz theorem, this uniform circulation along the wing length leads to a single vortex of the same strength trailing from each of the wing tips. This is a significant simplification of the problem and quantitative determination of finite wing aerodynamics calls for a more accurate model [15]. Thus, we find it necessary to further develop the approach by setting the circulation strength along the wing to an elliptic distribution which from lifting line theory is known to produce a constant downwash velocity distribution $[15,16]$. Since lift and hence bound circulation falls to zero at the inboard edge of the wing and there is no carryover of lift to the opposite wing $[9,17,18], \Gamma(r)$ should vary elliptically on a single wing spanning along $0 \leq r \leq R$. Therefore, $\Gamma(r)$ is expressed as an ellipse with the centre at $R / 2$ and a major axis length of $R$ as follows:

$\Gamma(\hat{r})=\Gamma_{\max } \sqrt{1-\left(2\left(\hat{r}-\frac{1}{2}\right)\right)^{2}} .(6)$

After simple manipulation it can be shown that:

$\Gamma(\hat{r})=2 \Gamma_{\max } \hat{r} \sqrt{\frac{1}{\hat{r}}-1}$.(7) 
Substituting equation 7 into equation 5 , it can be shown that the optimum chord distribution is proportional to the expression $\sqrt{\frac{1}{\hat{r}}-1}$. Thus, the optimum chord distribution for a revolving wing can be written as:

$c(\hat{r})=\frac{2.019 \bar{c}}{\pi} \sqrt{\frac{1}{\hat{r}}-1},(8)$

where $\bar{c}$ is the mean geometric chord and the factor $2.019 / \pi$ is obtained from satisfying the condition $\int_{0}^{1} \frac{c(\hat{r})}{\bar{c}} d \hat{r}=1$ [19]. This optimum chord distribution is shown in Fig. 1 together with a number of other relevant chord distributions for reference. Note that the obtained optimum chord distribution will only minimise induced losses due to downwash distribution effects; other sources of induced losses due to wake periodicity and effective actuator disk area are mainly functions of wing kinematics and are thus not accounted for.

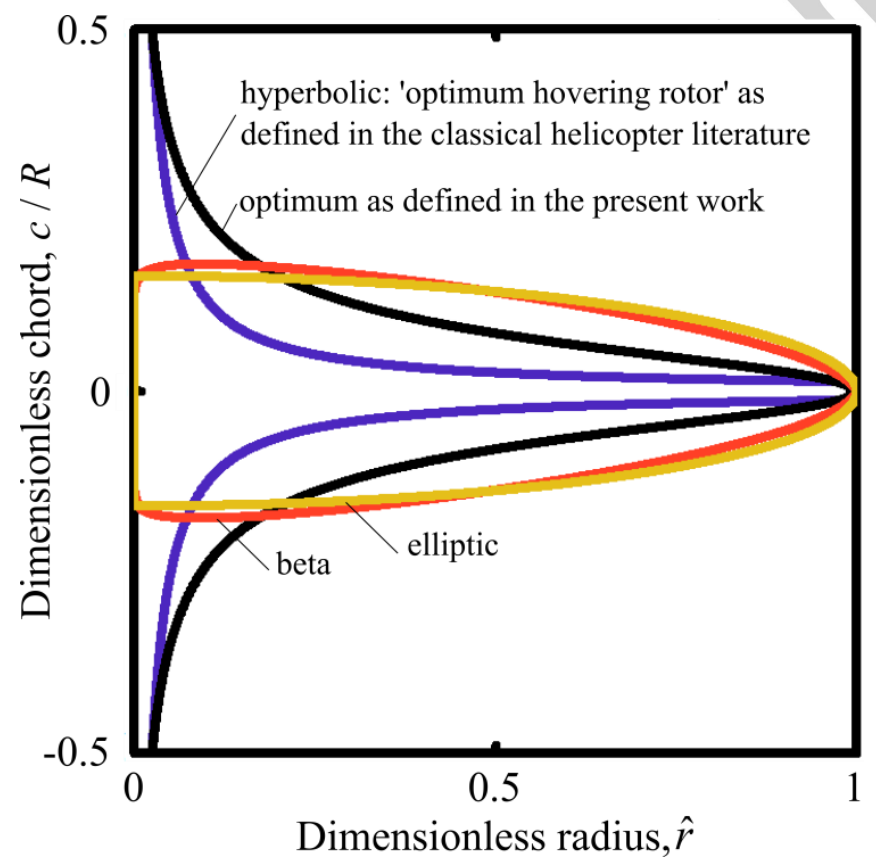

Figure 1: Comparison of the chord distribution for minimum total power for a revolving wing against a number of reference planforms. The so-called optimum distribution (black) is the chord distribution 
derived in this work (equation 8) and is capable of producing an elliptic circulation distribution on a revolving wing. The hyperbolic distribution (blue) produces a constant circulation distribution on a revolving wing. Within the helicopter literature, it has been referred to as the 'optimum hovering rotor' $[12,14]$. The beta distribution (red) is a practical representation of a hummingbird-like wing planform from biological studies $[19,20]$. In practice, it provides a good compromise between aerodynamic performance with respect to induced losses and other practical constraints. The elliptic distribution (yellow) produces an elliptic circulation distribution and hence a constant downwash distribution for parallel translating wings. For untwisted revolving wings, it produces a linear downwash distribution and thus a constant induced angle of attack along the wing length. In this illustration, all wings have an aspect ratio of 4 and same length $R$. For visualisation purposes, wings are shown symmetric about the wing centre line.

For a flapping wing, the forward and backward reciprocating motion implies a periodic change in sign of the implemented twist distribution which is mechanically expensive to implement within engineered insect-like designs except through passive aeroelastic means (as in real insect wings). Thus, we now consider the effect of designing for chord distribution only (zero twist) on the aerodynamic performance. By prescribing the chord distribution only, only one of the two optimality conditions can be achieved. First, we relax the constant downwash velocity constraint (minimum induced power) while maintaining operation at a constant effective angle of attack equal to the optimum wing section angle of attack along the wing length (minimum profile power). For an untwisted wing, a constant distribution of the effective angle of attack is obtained when a constant induced angle of attack distribution is achieved (see equations 1 and 2). In turn, this is obtained from a linear variation of the downwash which can be realised for revolving wings through the elliptic chord distribution [21]:

$c(\hat{r})=\frac{4 \bar{c}}{\pi} \sqrt{1-\hat{r}^{2}} \cdot(9)$

This shows that the elliptic chord distribution leads to a constant induced angle of attack distribution for both parallel and revolving translations, which is, to the authors at least, a delightful result. The constant sectional velocity distribution associated with parallel translation motion (fixed wing) leads in turn to a constant downwash velocity distribution and thus a unity induced power factor, where the induced power factor (denoted as $k_{\text {ind }}$ ) is the ratio of the actual induced power to minimum ideal induced power for a given lift [21-24]. On the other hand, the linear downwash distribution of the elliptic chord 
for a revolving wing leads to a $k_{\text {ind }}$ value of 1.13 (i.e. $13 \%$ more induced power compared to the ideal uniform downwash induced power to produce that lift $[21,22])$.

(a)

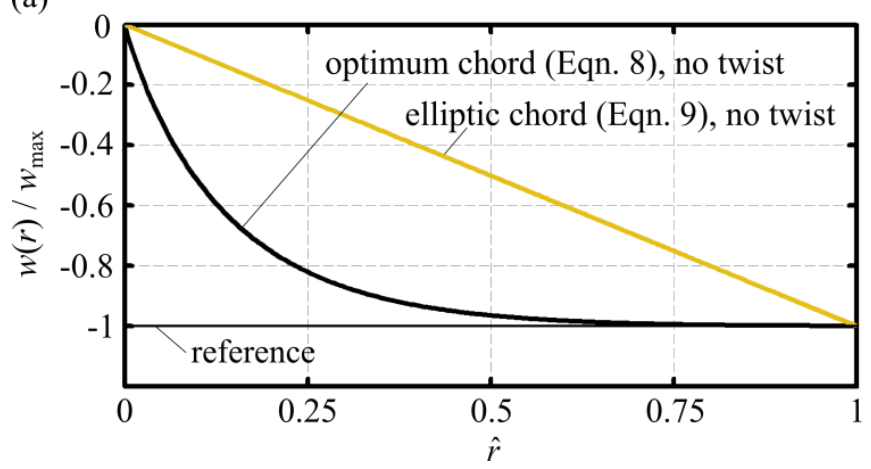

(b)

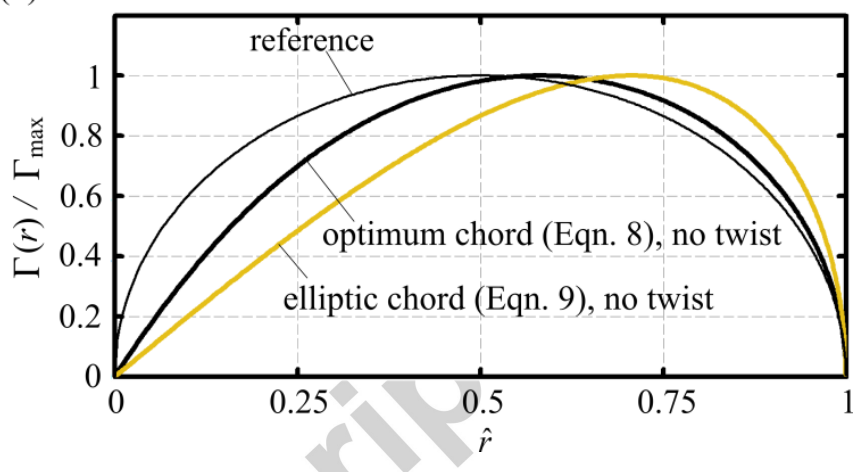

Figure 2: Effect of enforcing a no twist constraint on (a) downwash velocity and (b) circulation distributions for the chord distributions prescribed by equations 8 and 9 . In this illustration, the wing aspect ratio is 4 for the two chord distributions. An untwisted revolving wing with an elliptic chord distribution produces a linear downwash distribution. An untwisted revolving wing with the chord distribution prescribed by equation 8 produces a downwash distribution very near to the constant distribution and thus is still able to significantly reduce the induced power expenditure. The reference conditions (i.e. constant downwash and elliptic circulation distributions) are included to qualitatively correlate them to those of the two chords without twist. Downwash and circulation distributions are normalised using the maximum value of each distribution. Downwash and circulation distributions are evaluated based on the lifting line blade theory, for details see reference [21].

Next, if the constant effective angle of attack requirement is relaxed and no twist distribution is applied to the wing, it is found that the optimum chord distribution prescribed by equation 8 still significantly reduces the induced power expenditure. This is shown in Fig. 2 through plotting the downwash and circulation distributions for this chord distribution using the lifting line blade model in [21]. The downwash and circulation distributions produced are very near to the constant and elliptic distributions respectively. To further demonstrate this point, Fig. 3 shows the induced power factor, $k_{\text {ind }}$, for the untwisted optimum wing planform for different aspect ratios $(A R=R / \bar{c})$ within the practical operational range. The $k_{\text {ind }}$ values for the optimum chord shown in Fig. 3 slightly decrease with wing aspect ratio, and attain values of 1.016 for an aspect ratio of 3 and 1.003 for an aspect ratio of 7 . Figure 3 
also shows the variation of the induced power factor of the elliptic chord distribution which is found to be independent of aspect ratio. Whilst there are theoretically established aerodynamic benefits from increasing the aspect ratio such as increased maximum lift coefficient, and reduced induced losses [13], a high aspect ratio wing operating at high incidence is more prone to stall [5,6]. Of equal importance, higher aspect ratio leads to higher moment of inertia and hence higher inertial power cost.

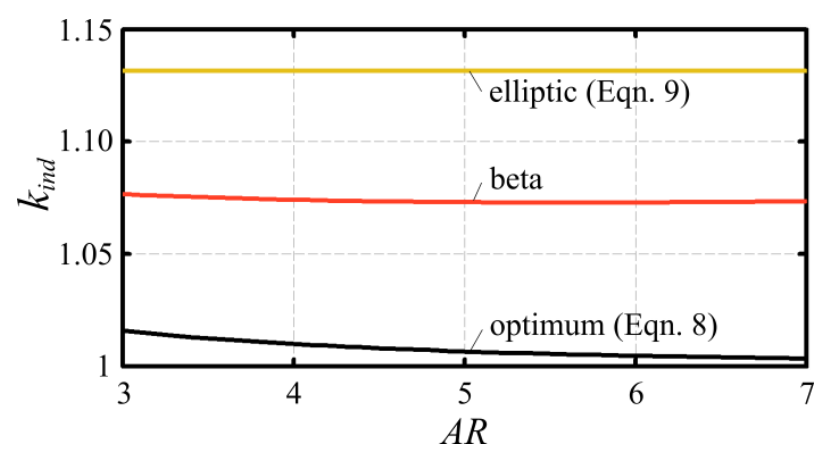

Figure 3: Variation of the induced power factor with wing aspect ratio. Wing twist is zero in each case. The value of $k_{\text {ind }}$ is evaluated based on the method developed in reference [21].

As a comment on practicality, the optimum chord distribution presented in this work is clearly difficult to implement due to the width of the root region. In a previous demonstration, we identified the 'arcsech' chord distribution as a possible solution for reducing $k_{\text {ind }}$ values [21]. The 'arcsech' chord distribution has relatively less root broadening compared to the optimum chord presented here, however it produces higher $k_{\text {ind }}$ values (the 'arcsech' distribution produces a $k_{\text {ind }}$ value of 1.036 for a wing with aspect ratio of 3 , and $k_{\text {ind }}$ decreases to a value of 1.016 for a wing with an aspect ratio of 7 ). A less optimal but more practical solution can be achieved by using a 'beta' chord distribution with an area centroid at $40 \%$ of the wing length [19,21] similar to a hummingbird wing planform [20] as introduced in Fig. 1. Again, the cost of increased practicality is an increase in the induced power factor which is raised to 1.07 , see Fig. 3 . The previously discussed cost only considers the translational aerodynamics aspect. 
From a structural perspective, it is noted that the broad root chord of the optimal wing planform would present an inertial penalty at stroke reversal for flapping, and this cost would have to be considered as part of a more integrated optimisation study. Another point to consider is that most animal wings are not symmetric around the wing centre line. The planform leading edge profile affects the wing rotational dynamics through its contribution to the aerodynamic moment around the wing spanwise axis [25]. As the current study does not consider rotational aerodynamics, this effect is ignored here.

In conclusion, an optimum revolving wing planform based on the elliptic circulation distribution has been derived. This represents a refinement to current practice in the literature where a simplified vortex model with a bound vortex of constant circulation is employed. Additionally, important insights into the aerodynamic performance of several untwisted revolving wing planforms are presented. It has been shown that even without applying any twist distribution the optimum chord distribution derived in this work produces a downwash distribution close to constant and thus the induced power expenditure is still significantly reduced. The optimum chord distribution requires a very broad root region; however a hummingbird-like wing planform provides a slightly less optimal performance in terms of induced power but with a more practical planform shape. The elliptic wing has always been an attractive planform known for its minimum induced drag within the fixed wing literature. The current study has demonstrated the unique aerodynamic advantage of the elliptic wing for a revolving motion. It has been shown that for any aspect ratio an untwisted elliptic revolving wing can allow all wing sections to operate at the same optimum effective angle of attack for a minimum profile power requirement.

\section{References:}

1. Ansari, S. A., Knowles, K., Zbikowski, R. 2008 Insectlike flapping wings in the hover part 2: Effect of wing geometry. J. Aircraft, 45, 1976-1990. (doi 10.2514/1.35697)

2. Usherwood, J. R., Ellington, C. P. 2002 The aerodynamics of revolving wings I. Model hawkmoth wings. $J$. Exp. Biol., 205, 1547-1564.

3. Usherwood, J. R., Ellington, C. P. 2002 The aerodynamics of revolving wings II. Propeller force coefficients from mayfly to quail. J. Exp. Biol., 205, 1565-1576.

4. Kruyt, J. W., Quicazán-Rubio, E. M., van Heijst, G. F., Altshuler, D. L., Lentink, D. 2014 Hummingbird wing efficacy depends on aspect ratio and compares with helicopter rotors. J. R. Soc. Interface 11: 20140585. (doi 10.1098/rsif.2014.0585) 
5. Kruyt, J. W., van Heijst, G. F., Altshuler, D. L., Lentink, D. 2015 Power reduction and the radial limit of stall delay in revolving wings of different aspect ratio. J. R. Soc. Interface 12: 20150051. (doi 10.1098/rsif.2015.0051)

6. Phillips, N., Knowles, K., Bomphrey, R. J. 2015 The effect of aspect ratio on the leading-edge vortex over an insect-like flapping wing. Bioinspir. Biomim. 10: 056020. (doi 10.1088/1748-3190/10/5/056020)

7. Harbig, R. R., Sheridan, J., Thompson, M. C. 2013 Reynolds number and aspect ratio effects on the leadingedge vortex for rotating insect wing planforms. J. Fluid Mech. 717, 166-192. (doi 10.1017/jfm.2012.565)

8. Dickinson, M. H., Lehmann, F. O., Sane, S. P. 1999 Wing rotation and the aerodynamic basis of insect flight. Science, 284, 1954-1960. (doi 10.1126/science.284.5422.1954)

9. Nabawy, M. R. A., Crowther, W. J. 2014 On the quasi-steady aerodynamics of normal hovering flight part II: model implementation and evaluation. J. R. Soc. Interface 11: 20131197. (doi 10.1098/rsif.2013.1197)

10. Nabawy, M. R. A., Crowther, W. J. 2015 Aero-optimum hovering kinematics. Bioinspir. Biomim. 10: 044002. (doi 10.1088/1748-3190/10/4/044002)

11. Gessow, A. 1948 Effect of rotor-blade twist and plan-form taper on helicopter hovering performance. NACA Technical Note No. 1542.

12. Gordon Leishman, J. 2006 Principles of Helicopter Aerodynamics, $2^{\text {nd }}$ ed., Cambridge University Press, Cambridge, Chapter 3.

13. Nabawy, M. R. A., Crowther, W. J. 2015 A quasi-steady lifting line theory for insect-like hovering flight. $P L o S$ ONE, 10: e0134972. (doi 10.1371/journal.pone.0134972)

14. Johnson, W. 1980 Helicopter Theory, Princeton University Press, Princeton, New Jersey, Chapter 2.

15. Schlichting, H., Truckenbrodt, E. 1979 Aerodynamics of the Airplane. McGraw-Hill, Inc, New York, Chapter 3.

16. Seddon, J. 1990 Basic helicopter aerodynamics.BSP Professional Books, Oxford, Chapter 2.

17. Nabawy, M. R. A., Crowther, W. J. Is flapping flight aerodynamically efficient? 32nd AIAA Applied Aerodynamics Conference, AIAA Aviation and Aeronautics Forum and Exposition, 16 - 20 June 2014, Atlanta, Georgia. (doi 10.2514/6.2014-2277)

18. Bomphery, R. J., Taylor, G. K., Thomas, A. L. R. 2009 Smoke visualization of free-flying bumblebees indicates independent leading edge vortices on each wing pair. Exp. Fluids 46, 811-821. (doi 10.1007/s00348-009-06318)

19. Ellington C.P. 1984 The aerodynamics of hovering insect flight: II. Morphological parameters. Phil. Trans. R. Soc. Lond. B 305, 17-40. (doi 10.1098/rstb.1984.0050)

20. Altshuler, D. L., Dudley, R., Ellington, C. P. 2004 Aerodynamic forces of revolving hummingbird wings and wing models. Journal of zoology, 264, 327-332. (doi 10.1017/S0952836904005813)

21. Nabawy, M. R. A., Crowther, W. J. 2014 On the quasi-steady aerodynamics of normal hovering flight part I: the induced power factor. J. R. Soc. Interface 11: 20131196. (doi 10.1098/rsif.2013.1196)

22. Ahmed, M. R., Abdelrahman, M. M., ElBayoumi, G. M., ElNomrossy, M. M. 2011 Optimal wing twist distribution for roll control of MAVs. Aeronaut. J. 115, 641- 649.

23. Henningsson, P., Bomphrey, R. J. 2012 Time-varying span efficiency through the wingbeat of desert Locust. J. R. Soc. Interface 9, 1177-1186. (doi 10.1098/rsif.2011.0749)

24. Henningsson, P., Bomphrey, R. J. 2013 Span efficiency in hawkmoths. J. R. Soc. Interface 10: 20130099. (doi 10.1098/rsif.2013.0099)

25. Whitney, J. P., Wood, R. J. 2010 Aeromechanics of passive rotation in flapping flight. J. Fluid Mech. 660, 197220. (doi 10.1017/S002211201000265X)

Highlights:

- A theoretical solution for the optimum hovering wing planform is identified.

- Aerodynamic performance of untwisted hovering wings is evaluated.

- An untwisted elliptical planform minimises profile power.

- An untwisted hummingbird-like planform minimises induced power. 